\title{
DISCUSSION
}

\section{THE SEISMIC RESISTANCE OF UNREINFORCED MASONRY CANTILEVER WALLS IN LOW SEIMICITY AREAS}

\author{
N.T.K. Lam, J.L. Wilson and G.L. Hutchinson, \\ vol 28, No 3, September 1995, pp179-195.
}

\section{A L ANDREWS}

Lam and co-authors should not have been, and probably were not, surprised by the failures they report of response spectrum methods to predict strengths of face loaded masonry walls. Familiar elastic response spectra do not describe masonry wall behaviour.

Simple harmonic motion (SHM) in single-degree-of-freedom systems has restoring forces proportional to displacements from the at rest configurations, and this property, together with earthquake motions and damping factors, gives definition to response spectra. Various empirical modifiers have been proposed to extend applicability of these spectra, principally to one defined kind of inelastic behaviour $[11,7]$, but also to a few bi-linearly elastic and inelastic behaviours [12]; people tempted to use this spectral information to assess kinetic energy must remember that pseudo-velocity information is not given reliably.

In contrast with the restoring force for an SHM system, which increases with displacement, the restoring force for a rocking system is greatest when the displacement from the rest position is infinitesimal, and it reduces curvilinearly with increasing displacement. Spectra for SHM systems are not applicable, and equations (7b) and (8) of the paper are not available discussion and observation based upon results of applying these and derived equations are suspect at best.

Although the authors are aware that the systems they investigated are not insulated from external energy sources and sinks, so the energy conservation principle needs to be handled very carefully, it seems that they have overlooked the influences of vertical acceleration (upon potential energy) and of radiation damping, since neither was mentioned. Both are likely to be important.

Finally, the authors cited reference 8 , results from which have also been used in recent work, now published in draft, by a study group of our Society. It needs to be recognised with regard to the reference 8 treatment of face loaded walls that the "equal-energy principle", invoked for obtaining spectral acceleration from displacement work done, is an empirical observation rather than a principle, and validated only for a very differently behaving class of systems. Therefore it must not be accepted in this application without specific justification, which it is unlikely to get.

\section{Additional references}

11. Veletsos A S and N M Newmark, 1960. Effect of Inelastic Behaviour on the Response of Simple Systems to Earthquake Motions, Proc. II WCEE, Tokyo.

12. Newmark N M and E Rosenblueth, 1971. Fundamentals of Earthquake Engineering, Prentice-Hall Inc..

\section{AUTHORS' REPLY}

The authors wish to thank A L Andrews for his comments on the paper and reply as follows :-

- Unreinforced masonry (URM) walls are traditionally regarded as a very brittle form of construction and for this reason elastic analysis based on the use of the response spectrum would still appear to many engineers as an appropriate method to estimate its response to earthquake loads, particularly in the out-of-plane direction. It was the purpose of the paper to highlight and demonstrate the shortcomings of the Response Spectrum Method and the Equal Energy Method in estimating the out-of-plane rocking response of a free-standing vertical cantilever wall.

- The authors recognise and agree that the equal energy method is based on an empirical observation (rather than a principle) which attempts to model complex non-linear behaviour using a simple linear elastic system. This study has reviewed the method and has highlighted the following two points :-

(a) It was shown using a non-linear time-history two examples (Cases $\mathrm{A}$ and $\mathrm{B}$, section 4.5.2) that the maximum velocity of the wall could be predicted from the elastic response spectrum with reasonable accuracy (despite the non-linear behaviour) provided that the rocking frequency associated with the maximum displacement was used in the calculation. Such rocking frequencies can be obtained experimentally (for example Figure 3) or from formulae such as those proposed by Yim et. al. [13].

(b) The method assumes zero energy input over the time period and does not account for the effects of acceleration pulses applied to a wall undergoing a large displacement (Case B, section 4.5.2). Recent analytical studies by the authors [14] demonstrate that the risk of overturning is significantly increased as the ground motion duration is increased (whilst the maximum acceleration and velocity are maintained constant).

- Vertical accelerations were outside the scope of this paper. 
- The effects of radiation damping were included as the damping constant used in the. analyses were calculated from experimental rocking tests on the shaking table.

- Further studies examining the rocking behaviour of a variety of cantilever walls have been carried out using an ensemble of synthetic and real earthquake records reflecting a wide range of frequency contents and durations [14].

\section{References:}

13. Yim, C.K., Chopra, A.K. and Penzien, J, 1980. Rocking Response of Rigid Blocks to Earthquakes, Earthquake Engineering and Structural Dynamics, 8:565-587.

14. Lam,N.T.K., Wilson,J.L. and Hutchinson, G.L., 1995. Rocking of Rigid Objects Subject to Base Excitation Using Time-History Analysis in Proceedings of the 14th Australasian Conference on the Mechanics of Structures and Materials, Hobart, Tasmania, 284-289. 